Article

\title{
No Uncountable Polish Group Can be a Right-Angled Artin Group
}

\author{
Gianluca Paolini ${ }^{1, *}$ and Saharon Shelah 1,2 \\ 1 Einstein Institute of Mathematics, The Hebrew University of Jerusalem, Edmond J. Safra Campus, \\ Givat Ram, Jerusalem 91904, Israel; shelah@math.huji.ac.il \\ 2 Department of Mathematics, The State University of New Jersey, Hill Center-Busch Campus, Rutgers, \\ 110 Frelinghuysen Road, Piscataway, NJ 08854-8019, USA \\ * Correspondence: gianluca.paolini@mail.huji.ac.il; Tel.: +972-2-658-4103
}

Academic Editor: Sidney A. Morris

Received: 28 March 2017; Accepted: 4 May 2017; Published: 11 May 2017

\begin{abstract}
We prove that if $G$ is a Polish group and $A$ a group admitting a system of generators whose associated length function satisfies: (i) if $0<k<\omega$, then $\lg (x) \leq \lg \left(x^{k}\right)$; (ii) if $\lg (y)<k<\omega$ and $x^{k}=y$, then $x=e$, then there exists a subgroup $G^{*}$ of $G$ of size $\mathfrak{b}$ (the bounding number) such that $G^{*}$ is not embeddable in $A$. In particular, we prove that the automorphism group of a countable structure cannot be an uncountable right-angled Artin group. This generalizes analogous results for free and free abelian uncountable groups.
\end{abstract}

Keywords: descriptive set theory; polish group topologies; right-angled Artin groups

In a meeting in Durham in 1997, Evans asked if an uncountable free group can be realized as the group of automorphisms of a countable structure. This was settled in the negative by Shelah [1]. Independently, in the context of descriptive set theory, Becher and Kechris [2] asked if an uncountable Polish group can be free. This was also answered negatively by Shelah [3], generalizing the techniques of [1]. Inspired by the question of Becher and Kechris, Solecki [4] proved that no uncountable Polish group can be free abelian. In this paper, we give a general framework for these results, proving that no uncountable Polish group can be a right-angled Artin group (see Definition 1). We actually prove more:

Theorem 1. Let $G=(G, d)$ be an uncountable Polish group and $A$ a group admitting a system of generators whose associated length function satisfies the following conditions:

(i) if $0<k<\omega$, then $\lg (x) \leq \lg \left(x^{k}\right)$;

(ii) if $\lg (y)<k<\omega$ and $x^{k}=y$, then $x=e$.

Then $G$ is not isomorphic to $A$; in fact, there exists a subgroup $G^{*}$ of $G$ of size $\mathfrak{b}$ (the bounding number) such that $G^{*}$ is not embeddable in $A$.

After the authors proved Theorem 1, they discovered that the impossibility to endow groups $A$ as in Theorem 1 with a Polish group topology follows from an old important result of Dudley [5]. In fact, Dudley's work implies more strongly that we cannot even find a homomorphism from a Polish group $G$ into A. Apart from the fact that the claim about $G^{*}$ in Theorem 1 is of independent interest and not subsumed by Dudley's work, our focus here is on techniques; i.e., the crucial use of the Compactness Lemma of [3]. This powerful result has a broad scope of applications, and is used by the authors in a work in preparation [6] to deal with classes of groups not covered by Theorem 1 or Dudley's work, most notably the class of right-angled Coxeter groups (see Definition 1).

Proof of Theorem 1. Let $\zeta=\left(\zeta_{n}\right)_{n<\omega} \in \mathbb{R}^{\omega}$ be such that $\zeta_{n}<2^{-n}$, for every $n<\omega$, and $\bar{g}=\left(g_{n}\right)_{n<\omega} \in G^{\omega}$ such that $g_{n} \neq e$ and $d\left(g_{n}, e\right)<\zeta_{n}$, for every $n<\omega$. Let $\Lambda$ be a set of power 
$\mathfrak{b}$ of increasing functions $\eta \in \omega^{\omega}$ which is unbounded with respect to the partial order of eventual domination. For transparency, we also assume that for every $\eta \in \Lambda$ we have $\eta(0)>0$. For $\eta \in \Lambda$, define the following set of equations:

$$
\Gamma_{\eta}=\left\{x_{n+1}^{\eta(n)}=x_{n} g_{n}: n<\omega\right\} .
$$

By (3.1, [3]), for every $\eta \in \Lambda, \Gamma_{\eta}$ is solvable in $G$. Let $\bar{b}_{\eta}=\left(b_{\eta, n}\right)_{n<\omega}$ witness it; i.e.,

$$
\bar{b}_{\eta} \in G^{\omega} \text { and } \bigwedge_{n<\omega} b_{\eta, n+1}^{\eta(n)}=b_{\eta, n} g_{n} .
$$

Let $G^{*}$ be the subgroup of $G$ generated by $\left\{g_{n}: n<\omega\right\} \cup\left\{b_{\eta, n}: \eta \in \Lambda, n<\omega\right\}$. Towards contradiction, suppose that $\pi$ is an embedding of $G^{*}$ into $A$, and let $S$ be a system of generators for $A$ whose associated length function $\lg _{S}=\lg$ satisfies conditions (i) and (ii) of the statement of the theorem. For $\eta \in \Lambda$ and $n<\omega$, let:

$$
\pi\left(g_{n}\right)=g_{n}^{\prime}, \pi\left(b_{\eta, n}\right)=c_{\eta, n} \text { and } m_{*}(\eta)=\lg \left(c_{\eta, 0}\right) .
$$

Now, $m_{*}$ is a function from $\Lambda$ to $\omega$ and so there exists unbounded $\Lambda_{1} \subseteq \Lambda$ such that for every $\eta \in \Lambda_{1}$ the value $m_{*}(\eta)$ is a constant $m_{*}$. Fix such a $\Lambda_{1}$ and $m_{*}$, and let $f_{1}, f_{2} \in \omega^{\omega}$ increasing satisfying the following:

(1) $f_{1}(n)>\lg \left(g_{n}^{\prime}\right)$;

(2) $f_{2}(n)=\left(m_{*}+1\right)+\sum_{\ell<n} f_{1}(\ell)$.

Claim 1. For every $\eta \in \Lambda_{1}, \lg \left(c_{\eta, n}\right)<f_{2}(n)$.

Proof. By induction on $n<\omega$. The case $n=0$ is clear by the choice of $f_{1}$ and $f_{2}$. Let $n=m+1$. Because of assumption (i) on $A$, the choice of $\Lambda_{1}$, and the choice of $f_{1}$ and $f_{2}$, we have:

$$
\begin{aligned}
\lg \left(c_{\eta, n}\right) & \leq \lg \left(c_{\eta, n}^{\eta(m)}\right) \\
& =\lg \left(c_{\eta, m} g_{m}^{\prime}\right) \\
& \leq \lg \left(c_{\eta, m}\right)+\lg \left(g_{m}^{\prime}\right) \\
& <f_{2}(m)+f_{1}(m) \\
& =f_{2}(n) .
\end{aligned}
$$

Now, by the choice of $\Lambda_{1}$, we can find $\eta \in \Lambda_{1}$ and $n<\omega$ such that $\eta(n)>f_{2}(n+2)$. Notice then that by the claim above and the choice of $f_{1}$ and $f_{2}$, we have:

$$
\begin{gathered}
\eta(n)>f_{2}(n+1)=f_{2}(n)+f_{1}(n)>\lg \left(c_{\eta, n}\right)+\lg \left(g_{n}^{\prime}\right) \geq \lg \left(c_{\eta, n} g_{n}^{\prime}\right), \\
\eta(n)>f_{2}(n+2) \geq f_{1}(n+1)>\lg \left(g_{n+1}^{\prime}\right) .
\end{gathered}
$$

Thus, by (1) and the fact that $c_{\eta, n+1}^{\eta(n)}=c_{\eta, n} g_{n}^{\prime}$, using assumption (ii), we infer that $c_{\eta, n+1}=e$. Hence,

$$
c_{\eta, n+2}^{\eta(n+1)}=c_{\eta, n+1} g_{n+1}^{\prime}=g_{n+1}^{\prime}
$$

Furthermore, if $\eta(n+1)>\lg \left(g_{n+1}^{\prime}\right)$, then again by assumption (ii), we have that $c_{\eta, n+2}=e$, and so $c_{\eta, n+2}^{\eta(n+1)}=g_{n+1}^{\prime}=e$, which contradicts the choice of $\left(g_{n}\right)_{n<\omega}$. Hence, $\eta(n)<\eta(n+1) \leq \lg \left(g_{n+1}^{\prime}\right)$, contradicting (2). It follows that the embedding $\pi$ from $G^{*}$ into $A$ cannot exist. 
Definition 1. Given a graph $\Gamma=(E, V)$, the right-angled Artin group $A(\Gamma)$ is the group with presentation:

$$
\Omega(\Gamma)=\langle V \mid a b=b a: a E b\rangle .
$$

If in the presentation $\Omega(\Gamma)$, we ask in addition that all the generators are involutions, then we speak of right-angled Coxeter groups $C(\Gamma)$.

Thus, for $\Gamma$, a graph with no edges (resp. a complete graph) $A(\Gamma)$ is a free group (resp. a free abelian group).

Definition 2. Let $A(\Gamma)$ be a right-angled Artin group and $\mathrm{lg}$ its associated length function. We say that an element $g \in A(\Gamma)$ is cyclically reduced if it cannot be written as $g=h f h^{-1}$ with $\lg (g)=\lg (f)+2$.

Fact 1. Let $A(\Gamma)$ be a right-angled Artin group, lg its associated length function, and $g \in A(\Gamma)$. Then:

(1) $g$ can be written as $h f h^{-1}$ with $f$ cyclically reduced and $\lg (g)=\lg (f)+2 \lg (h)$;

(2) if $0<k<\omega$ and $f$ is cyclically reduced, then $\lg \left(f^{k}\right)=k \lg (f)$;

(3) if $0<k<\omega$ and $g=h f h^{-1}$ is as in (1), then $\lg \left(h f h^{-1}\right)^{k}=k \lg (f)+2 \lg (h)$.

Proof. Item (1) is proved in (Proposition on p. 38, [7]). The rest is folklore.

Corollary 1. No uncountable Polish group can be a right-angled Artin group.

Proof. By Theorem 1 it suffices to show that for every right-angled Artin group $A(\Gamma)$ the associated length function $\lg$ satisfies conditions (i) and (ii) of the theorem, but by Fact 1, this is clear.

As is well known, the automorphism group of a countable structure is naturally endowed with a Polish topology which respects the group structure, hence:

Corollary 2. The automorphism group of a countable structure cannot be an uncountable right-angled Artin group.

As already mentioned, the situation is different for right-angled Coxeter groups; in fact, the structure $M$ with $\omega$ many disjoint unary predicates of size 2 is such that $\operatorname{Aut}(M)=\left(\mathbb{Z}_{2}\right)^{\omega}$; i.e., $\operatorname{Aut}(M)$ is the right-angled Coxeter group on $K_{\mathfrak{c}}$ (a complete graph on continuum many vertices). Notice that in this group for any $a \neq b \in K_{\mathfrak{c}}$, we have:

(i) $(a b)^{2}=1$;

(ii) $\lg (a b)=2<3,(a b)^{3}=a b$ and $a b \neq e$.

Acknowledgments: Partially supported by European Research Council grant 338821. No. 1112 on Shelah's publication list.

Author Contributions: Gianluca Paolini and Saharon Shelah contribute equally to this manuscript.

Conflicts of Interest: The authors declare no conflict of interest.

\section{References}

1. Shelah, S. A Countable Structure Does Not Have a Free Uncountable Automorphism Group. Bull. Lond. Math. Soc. 2003, 35, 1-7.

2. Becker, H.; Kechris, A.S. The Descriptive Set Theory of Polish Group Actions; London Math. Soc. Lecture Notes Ser. 232; Cambridge University Press: Cambridge, UK, 1996.

3. Shelah, S. Polish Algebras, Shy From Freedom. Israel J. Math. 2011, 181, 477-507.

4. Solecki, S. Polish Group Topologies. In Sets and Proofs; London Math. Soc. Lecture Note Ser. 258; Cambridge University Press: Cambridge, UK, 1999. 
5. Dudley, R.M. Continuity of Homomorphisms. Duke Math. J. 1961, 28, 34-60.

6. Paolini, G.; Shelah, S. Polish Topologies for Graph Products of Cyclic Groups. In Preparation.

7. Servatius, H. Automorphisms of Graph Groups. J. Algebra 1989, 126, 34-60.

(C) 2017 by the authors. Licensee MDPI, Basel, Switzerland. This article is an open access article distributed under the terms and conditions of the Creative Commons Attribution (CC BY) license (http:// creativecommons.org/licenses/by/4.0/). 